\title{
Bounds on norms of compound matrices and on products of eigenvalues *
}

\author{
Ludwig Elsner \\ Fakultät für Mathematik \\ Universität Bielefeld \\ Postfach 100131 \\ D-33615 Bielefeld \\ Germany
}

\author{
Daniel Hershkowitz \\ Department of Mathematics \\ Technion - Israel Institute of Technology \\ Haifa 32000 \\ Israel
}

\author{
Hans Schneider \\ Department of Mathematics \\ University of Wisconsin \\ Madison, Wisconsin 53706 \\ U.S.A
}

21 November 1997

\begin{abstract}
An upper bound on operator norms of compound matrices is presented, and special cases that involve the $l_{1}, l_{2}$ and $l_{\infty}$ norms are investigated. The results are then used to obtain bounds on products of the largest or smallest eigenvalues of a matrix.
\end{abstract}

*The research was started while the first author visited the Technion in 1995. It was continued during a visit of the second author at Universität Bielefeld and at the University of Wisconsin - Madison in 1997, and it was completed during a visit of the third author at the Technion in 1997. The research was partly supported by SFB 343 "Diskrete Strukturen in der Mathematik", Bielefeld, and by NSF Grant DMS-9424346. 


\section{Introduction}

Let $A$ be a complex matrix and let $C_{k}(A)$ be its $k$ th compound. It was shown in [10, Formula (12)] that the maximal row sum (of moduli) of elements of $C_{k}(A)$ is less than or equal to the product of the $k$ largest rows sums of $A$, and it follows that the product of $k$ largest (moduli of) eigenvalues of $A$ is bounded above by the product of the $k$ largest row sums of $A$. The case of equality in these inequalities investigated in 9 , Theorems I and II].

The results in [10] and [9] can be viewed as relating the $l_{1}$ norm of rows of a matrix to the $l_{1}$ norm of its compounds (viewed as an operator on rows). Working in terms of columns, we consider in this paper the relations between other norms $\nu$ of columns and norms $\mu$ of the compounds. We begin by proving a general result of the above type which involves a constant $\theta_{k}(\mu, \nu)$. We evaluate this constant in some special cases that involve the $l_{1}, l_{2}$ and $l_{\infty}$ norms. Again, this leads naturally to upper bounds on the product of the $k$ largest eigenvalues or, equivalently, lower bounds on the product of the $k$ smallest eigenvalues, which involve products of norms of columns and of norms of rows of the matrix.

As a consequence of our theorems, we obtain generalizations of results of [4, [1] and 12] on bounds on norms of the adjoint matrix, which is essentially the $n-1$ compound matrix, to $k$ th compound matrices. The application of our theorems to the adjoint case sharpens the results in [4], [11] and [12].

\section{Upper bounds on norms of compound matrices}

Let $A$ be a matrix in $\mathbb{C}^{n n}$. For subsets $\alpha$ and $\beta$ of $\{1, \ldots, n\}$ we denote by $A(\alpha \mid \beta)$ the submatrix of $A$ whose rows are indexed by $\alpha$ and whose columns are indexed by $\beta$ in their natural order.

Let $k$ be a positive integer, $k \leq n$. We denote by $C_{k}(A)$ the $k$ th compound of the matrix $A$, that is, the $\left(\begin{array}{l}n \\ k\end{array}\right) \times\left(\begin{array}{l}n \\ k\end{array}\right)$ matrix whose elements are the minors $\operatorname{det} A(\alpha \mid \beta)$, $\alpha, \beta \subseteq\{1, \ldots, n\},|\alpha|=|\beta|=k$. We index $C_{k}(A)$ by $\alpha \subseteq\{1, \ldots, n\},|\alpha|=k$ (ordered lexicographically).

Let $\nu$ be a vector norm on $\mathbb{C}^{n}$, and for a positive integer $k, k \leq n$, let $\mu$ be a (submultiplicative) norm on $\mathbb{C}^{m m}$ where $m=\left(\begin{array}{l}n \\ k\end{array}\right)$. We define

$$
\theta_{k}(\mu, \nu)=\max \left\{\mu\left(C_{k}(B)\right): B \in \mathbb{C}^{n n}, \nu\left(\operatorname{col}_{i}(B)\right)=1, i=1, \ldots, n\right\},
$$

where $\operatorname{col}_{i}(B)$ denotes the $i$ th column of $B$.

The following theorem is the main tool from which we derive our results. 
Theorem 2.1 For an absolute operator norm $\mu$ we have

$$
\mu\left(C_{k}(A)\right) \leq \theta_{k}(\mu, \nu) \max _{\substack{\alpha \subseteq\{1, \ldots, n\} \\|\alpha|=k}} \prod_{i \in \alpha} \nu\left(\operatorname{col}_{i}(A)\right) .
$$

Proof. Assume first that $A$ has no zero columns. Note that for every nonsingular matrix $R$ we have

$$
C_{k}\left(A R^{-1}\right) C_{k}(R)=C_{k}(A) .
$$

In particular, if we choose the matrix $R$ to be the diagonal matrix $\operatorname{diag}\left(r_{i}\right)$ where $r_{i}=$ $\nu\left(\operatorname{col}_{i}(A)\right), i=1, \ldots, n$, then $C_{k}(R)$ is a diagonal matrix with diagonal elements $\prod_{i \in \alpha} r_{i}$, $\alpha \subseteq\{1, \ldots, n\},|\alpha|=k$. Since $\mu$ is an absolute operator norm, it follows by Theorem 3 in [2], see also [6, p.310, Theorem 5.6.37], that

$$
\mu\left(C_{k}(R)\right)=\max _{\substack{\alpha \subseteq\{1, \ldots, n\} \\|\alpha|=k}} \prod_{i \in \alpha} r_{i} .
$$

Since the $\nu$ norm of the columns of $A R^{-1}$ are equal to 1 , it now follows that

$$
\mu\left(C_{k}(A)\right) \leq \mu\left(C_{k}\left(A R^{-1}\right)\right) \mu\left(C_{k}(R)\right) \leq \theta_{k}(\mu, \nu) \max _{\substack{\alpha \subseteq\{1, \ldots, n\} \\|\alpha|=k}} \prod_{i \in \alpha} \nu\left(\operatorname{col}_{i}(A)\right) .
$$

If $A$ has zero columns then we apply the above procedure to the matrix $A+\epsilon I$ for $\epsilon$ sufficiently small, and then use continuity arguments to prove our assertion.

Remark 2.3 Note that inequality (2.2) is sharp since, by definition of $\theta_{k}(\mu, \nu)$, equality is attained for matrices whose columns have $\nu$ norm 1 .

In the rest of this section we evaluate $\theta_{k}(\mu, \nu)$ for cases that involve the $l_{p}$ norms, $p=1,2, \infty$. We denote these by $\|.\|_{p}$, and use the same notation for both vector norms and matrix operator norms.

The following proposition is proven in [9, Formulas (9) and (10)]. The inequality (2.5) also occurs in the proof of Theorem 1 in [10].

Proposition 2.4 For every $\beta \in\{1, \ldots, n\},|\beta|=k$ we have

$$
\| \operatorname{col}_{\beta}\left(C_{k}(A)\left\|_{1} \leq \max _{\substack{\alpha \subseteq\{1, \ldots, n\} \\|\alpha|=k}} \prod_{i \in \alpha}\right\| \operatorname{col}_{i}(A) \|_{1} .\right.
$$

Furthermore, if $A$ has at least $k$ nonzero columns then for a set $\beta \in\{1, \ldots, n\},|\beta|=k$, the following are equivalent:

(i) Equality holds in (2.5).

(ii) We have

$$
\prod_{i \in \beta}\left\|\operatorname{col}_{i}(A)\right\|_{1}=\max _{\substack{\alpha \subseteq\{1, \ldots, n\} \\|\alpha|=k}} \prod_{i \in \alpha}\left\|\operatorname{col}_{i}(A)\right\|_{1},
$$

and the columns of $A$ indexed by $\beta$ have disjoint supports. 
Definition 2.6 A matrix $A$ is said to be a monomial matrix if $A=P D$, where $P$ is a permutation matrix and $D$ is a diagonal matrix.

Theorem 2.7 We have $\theta_{k}\left(l_{1}, l_{1}\right)=1$.

Proof. Since

$$
\left\|C_{k}(A)\right\|_{1}=\max _{\substack{\beta \subseteq\{1, \ldots, n\} \\|\beta|=k}}\left\|\operatorname{col}_{\beta}\left(C_{k}(A)\right)\right\|_{1}
$$

it follows immediately from Proposition 2.4 that $\theta_{k}\left(l_{1}, l_{1}\right) \leq 1$. By Proposition 2.4, every monomial matrix $A$ satisfies

$$
\left\|C_{k}(A)\right\|_{1}=\max _{\substack{\beta \subseteq\{1, \ldots, n\} \\|\beta|=k}}\left\|\operatorname{col}_{\beta}\left(C_{k}(A)\right)\right\|_{1}=\max _{\substack{\alpha \subseteq\{1, \ldots, n\} \\|\alpha|=k}} \prod_{i \in \alpha}\left\|\operatorname{col}_{i}(A)\right\|_{1} .
$$

implying that $\theta_{k}\left(l_{1}, l_{1}\right)=1$.

Theorem 2.8 We have $\theta_{k}\left(l_{2}, l_{2}\right)=\left(\frac{n}{k}\right)^{\frac{k}{2}}$. Furthemore, if $A$ is nonsingular and $k<n$ then

$$
\left\|C_{k}(A)\right\|_{2}<\left(\frac{n}{k}\right)^{\frac{k}{2}} \max _{\substack{\alpha \subseteq\{1, \ldots, n\} \\|\alpha|=k}} \prod_{i \in \alpha}\left\|\operatorname{col}_{i}(A)\right\|_{2} .
$$

Proof. Let $A \in \mathbb{C}^{n n}$ be such that $\left\|\operatorname{col}_{i}(A)\right\|_{2}=1, i=1, \ldots, n$. The matrix $B=A^{*} A$ is positive semidefinite with diagonal entries equal to 1 . Let $\rho_{1} \geq \rho_{2} \geq \ldots \rho_{n} \geq 0$ be the eigenvalues of $B$. Note that $\rho_{1}+\ldots+\rho_{n}=\operatorname{trace}(B)=n$. It now follows that

$$
\left(\left\|C_{k}(A)\right\|_{2}\right)^{2}=\left\|C_{k}(B)\right\|_{2}=\rho_{1} \rho_{2} \cdots \rho_{k} \leq\left(\frac{\rho_{1}+\ldots+\rho_{k}}{k}\right)^{k} \leq\left(\frac{n}{k}\right)^{k} .
$$

We thus have

$$
\theta_{k}\left(l_{2}, l_{2}\right) \leq\left(\frac{n}{k}\right)^{\frac{k}{2}}
$$

We now prove that equality holds in (2.11). By [5], see also [8, Theorem 2], there exists a positive semidefinite $n \times n$ matrix $B$ with diagonal elements all equal to 1 and where the eigenvalues of $B$ are $\frac{n}{k}$ with multiplicity $k$ and 0 with multiplicity $n-k$. We have

$$
\left\|C_{k}(B)\right\|_{2}=\left(\frac{n}{k}\right)^{k}
$$

Now, let $A$ be the positive semidefinite matrix such that $B=A^{2}$. Since the diagonal entries of $B$ are all equal to 1 , it follows that $\left\|\operatorname{col}_{i}(A)\right\|_{2}=1, i=1, \ldots, n$. Also,

$$
\left\|C_{k}(A)\right\|_{2}=\sqrt{\left\|C_{k}(B)\right\|_{2}}=\left(\frac{n}{k}\right)^{\frac{k}{2}}
$$


proving that equality holds in (2.11). Finally, notice that if $A$ is nonsingular then $\rho_{n}>0$ and so strict inequality holds in (2.10) whenever $k<n$. Therefore, equality in (2.11) cannot be attained for nonsingular matrices, and using the techniques of the proof of Theorem 2.1 one can prove the strict inequality (2.9) whenever $A$ is nonsingular and $k<n$.

We remark that the inequality (2.11) in the case $k=n-1$ is proven in 12 , Theorem 4], see also [4, Lemma 2]. Our proof of this inequality is essentially the same as in [12

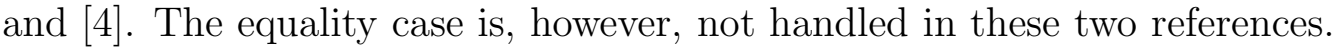

Theorem 2.12 For $k<n$ we have

$$
\theta_{k}\left(l_{\infty}, l_{\infty}\right) \leq\left(\begin{array}{l}
n \\
k
\end{array}\right)(k+1)^{\frac{k-1}{2}}
$$

Proof. Let $A \in \mathbb{C}^{n n}$ be such that $\left\|\operatorname{col}_{i}(A)\right\|_{\infty}=1, i=1, \ldots, n$. Let $x$ be a vector in $\mathbb{C}^{\left(\begin{array}{l}n \\ k\end{array}\right)}$ and let $y=C_{k}(A) x$. For every subset $\alpha$ of $\{1, \ldots n\}$ of cardinality $k$ we have

$$
y_{\alpha}=\sum_{\substack{\beta \subseteq\{1, \ldots, n\} \\|\beta|=k}} \operatorname{det} A(\alpha \mid \beta) x_{\beta} .
$$

We have $k<n$. Therefore, note that each subset $\beta$ of $\{1, \ldots n\}$ of cardinality $k$ is contained in $n-k$ different subsets $\gamma$ of $\{1, \ldots n\}$ of cardinality $k+1$. Therefore, we have

$$
\sum_{\substack{\beta \subseteq\{1, \ldots, n\} \\|\beta|=k}} \operatorname{det} A(\alpha \mid \beta) x_{\beta}=\frac{1}{n-k} \sum_{\substack{\gamma \subseteq\{1, \ldots, n\} \\|\gamma|=k+1}} \sum_{\substack{\beta \subseteq \gamma \\|\beta|=k}} \operatorname{det} A(\alpha \mid \beta) x_{\beta} .
$$

Observe that the rightmost sum of (2.15) is the determinant of the $(k+1) \times(k+1)$ matrix $B$ obtained by appending the subvector of $x$ (with possible different signs of elements) indexed by the subsets $\beta$ of $\gamma$ of cardinality $k$ as a row to the matrix $A(\alpha \mid \gamma)$. Thus, if we choose $x$ such that $\|x\|_{\infty}=1$ then the matrix $B$ has entries of modulus less than or equal to 1, and by the Hadamard determinant theorem, e.g. [7, p.114, Theorem 4.1.7] it follows that $\operatorname{det} B \leq \sqrt{k+1}^{k+1}$. Hence, it follows from (2.14) and (2.15) that

$$
\left\|C_{k}(A)\right\|_{\infty} \leq \frac{\left(\begin{array}{c}
n \\
k+1
\end{array}\right) \sqrt{k+1}^{k+1}}{n-k}=\left(\begin{array}{l}
n \\
k
\end{array}\right)(k+1)^{\frac{k-1}{2}},
$$

proving our assertion.

Note that $C_{n}(A)=\operatorname{det}(A)$. Therefore, in the case $k=n$ the Hadamard determinant theorem yields the following.

Theorem 2.16 We have

$$
\theta_{n}\left(l_{\infty}, l_{\infty}\right)=\sqrt{n}^{n} .
$$


In the cases $k=1$ and $k=n-1$ we have equality in (2.13) as follows.

Theorem 2.17 We have $\theta_{1}\left(l_{\infty}, l_{\infty}\right)=n$.

Proof. In view of the inequality (2.13) all we have to show is that there exists an $n \times n$ matrix $A$ satisfying $\left\|\operatorname{col}_{i}(A)\right\|_{\infty}=1, i=1, \ldots, n$ and such that $\|A\|_{\infty}=n$. It is easy to check that the $n \times n$ matrix whose first row consists of 1's and all other entries equal to 0 is such a matrix.

In order to establish the case $k=n-1$ we first make an observation.

Observation 2.18 For every positive integer $n$ there exists an $n \times n$ complex matrix $A$ satisfying $\left|a_{i j}\right|=1, i, j=1, \ldots, n$ and $A A^{*}=n I$. An example of such a matrix is the Vandermonde matrix

$$
\left(\begin{array}{ccccc}
1 & 1 & 1 & \ldots & 1 \\
1 & \omega & \omega^{2} & \ldots & \omega^{n-1} \\
1 & \omega^{2} & \omega^{4} & \ldots & \omega^{2 n-2} \\
\vdots & \vdots & \vdots & & \vdots \\
1 & \omega^{n-1} & \omega^{2 n-2} & \ldots & \omega^{(n-1)^{2}}
\end{array}\right)
$$

where $\omega=e^{\frac{2 \pi i}{n}}$. There are also the Hadamard matrices for those $n$ 's for which they exist.

Theorem 2.19 We have $\theta_{n-1}\left(l_{\infty}, l_{\infty}\right)=\sqrt{n}^{n}$.

Proof. In view of the inequality (2.13) all we have to show is that there exists an $n \times n$ matrix $A$ satisfying $\left\|\operatorname{col}_{i}(A)\right\|_{\infty}=1, i=1, \ldots, n$ and such that $\left\|C_{n-1} A\right\|_{\infty}=\sqrt{n}^{n}$. Let A be an $n \times n$ complex matrix satisfying $\left|a_{i j}\right|=1, i, j=1, \ldots, n$ and $A A^{*}=n I$. Then $A^{-1}=\frac{1}{n} A^{*}$, and so

$$
C_{n-1}(A)=\left(\operatorname{det}(A) D A^{-1} D\right)^{T}=\frac{\operatorname{det}(A)}{n} D \bar{A} D
$$

where $D$ is the diagonal matrix with alternating 1 's and -1 's along the diagonal. It now follows that $\left\|C_{n-1}(A)\right\|_{\infty}=\sqrt{n}^{n}$, proving our claim.

In order to consider some other combinations of norms, for a real number $r$ we denote $[r]^{+}=\max \{r, 0\}$.

Lemma 2.20 Let $\mu$ be an absolute operator norm and let $p$ and $r$ be positive integers. Then

$$
\theta_{k}\left(\mu, l_{p}\right) \leq n^{\left[\frac{k}{r}-\frac{k}{p}\right]^{+}} \theta_{k}\left(\mu, l_{r}\right)
$$


Proof. By [3, p.26 \#16 and p.29 \#19], for every vector $v$ in $\mathbb{C}^{n}$ we have

$$
\|v\|_{r} \leq n^{\left[\frac{1}{r}-\frac{1}{p}\right]^{+}}\|v\|_{p} .
$$

Our claim now follows from Theorem 2.1 and from the fact that (2.2) is sharp.

From Theorems 2.7, 2.8 and 2.12 we obtain, by Lemma 2.20, the following corollary.

Corollary 2.22 We have

and

$$
\begin{gathered}
\theta_{k}\left(l_{1}, l_{2}\right) \leq \sqrt{n}^{k}, \\
\theta_{k}\left(l_{1}, l_{\infty}\right) \leq n^{k}, \\
\theta_{k}\left(l_{2}, l_{1}\right) \leq\left(\frac{n}{k}\right)^{\frac{k}{2}}, \\
\theta_{k}\left(l_{2}, l_{\infty}\right) \leq\left(\frac{n}{k}\right)^{\frac{k}{2}} \sqrt{n}^{k}, \\
\theta_{k}\left(l_{\infty}, l_{1}\right) \leq \begin{cases}\left(\begin{array}{l}
n \\
k
\end{array}\right)(k+1)^{\frac{k-1}{2}}, & k<n \\
\sqrt{n}^{n}, & k=n\end{cases}
\end{gathered}
$$

(and

We conclude this section with two general remarks.

Remark 2.29 Note that one can define $\theta_{k}(\mu, \nu)$ using rows instead of columns and obtain similar results where rows replace columns all over.

Remark 2.30 Denote by $\operatorname{adj}(A)$ the (classical) adjoint matrix of $A$, that is, the transposed matrix of cofactors. Note that the term adjugate is sometimes used instead of adjoint to avoid confusion with the Hermitian adjoint $A^{*}$. Since $\operatorname{adj}(A)=D C_{n-1}\left(A^{T}\right) D$ where $D$ is the diagonal matrix with alternating 1's and -1 's along the diagonal, it follows that for absolute norms our results in the case $k=n-1$ yield an upper bound on $\mu(\operatorname{adj}(A))$. In particular, our remark applies to the $l_{1}, l_{2}$ and $l_{\infty}$ norms under discussion. Our results can also be applied to the adjoint compounds found in [1, Chapter 5]. 


\section{Bounds on products of eigenvalues}

For an $n \times n$ complex matrix $A$ we denote by $\lambda_{1}(A), \ldots, \lambda_{n}(A)$ the eigenvalues of $A$ ordered in a non-increasing order of their moduli. In this section we find an upper bound on the product $\left|\prod_{i=1}^{k} \lambda_{i}(A)\right|$ or, equivalently, a lower bound on the product $\left|\prod_{i=k+1}^{n} \lambda_{i}(A)\right|$.

Our results follow from the following corollary of Theorem 2.1. Here we denote by $\operatorname{row}_{i}(A)$ the (vector in $\mathbb{C}^{n}$ which is the) transpose of the $i$ th row of $A$.

Theorem 3.1 Let $\mu$ be an absolute operator norm on $\mathbb{C}^{m m}$ where $m=\left(\begin{array}{l}n \\ k\end{array}\right)$. Then

$$
\left|\prod_{i=1}^{k} \lambda_{i}(A)\right| \leq \theta_{k}(\mu, \nu) \min \left\{\max _{\substack{\alpha \subseteq\{1, \ldots, n\} \\|\alpha|=k}} \prod_{i \in \alpha} \nu\left(\operatorname{col}_{i}(A)\right), \max _{\substack{\alpha \subseteq\{1, \ldots, n\} \\|\alpha|=k}} \prod_{i \in \alpha} \nu\left(\operatorname{row}_{i}(A)\right)\right\} .
$$

Proof. As is well known, the spectral radius $\rho\left(C_{k}(A)\right)$ of $C_{k}(A)$ satisfies

$$
\rho\left(C_{k}(A)\right)=\left|\prod_{i=1}^{k} \lambda_{i}(A)\right| .
$$

Also, we have $\rho\left(C_{k}(A)\right)=\rho\left(C_{k}\left(A^{T}\right)\right)$. Since $\mu$ is an operator norm we have

$$
\rho\left(C_{k}(A)\right) \leq \mu\left(C_{k}(A)\right),
$$

Our claim follows from (3.2), (3.3) and (2.2), where the latter is applied both to $A$ and $A^{T}$.

It follows from Theorem 3.1 that in order to obtain a better upper bound on $\left|\prod_{i=1}^{k} \lambda_{i}(A)\right|$ in terms of the $\nu$ norms of the rows and columns of $A$, we should pick us the $\mu$ norm that provides the lowest value of $\theta_{k}(\mu, \nu)$. We now apply this approach to the results of the previous section. The best upper bound in terms of the $l_{1}$ norms of rows and columns that can be derived from Theorem 2.7 and from the inequalities (2.25) and (2.27) is

Theorem 3.4 Let $A \in \mathbb{C}^{n n}$. Then

$$
\left|\prod_{i=1}^{k} \lambda_{i}(A)\right| \leq \min \left\{\max _{\substack{\alpha \subseteq\{1, \ldots, n\} \\|\alpha|=k}} \prod_{i \in \alpha}\left\|\operatorname{col}_{i}(A)\right\|_{1}, \max _{\substack{\alpha \subseteq\{1, \ldots, n\} \\|\alpha|=k}} \prod_{i \in \alpha}\left\|\operatorname{row}_{i}(A)\right\|_{1}\right\} .
$$

Remark 3.6 The upper bound on $\left|\prod_{i=1}^{k} \lambda_{i}(A)\right|$ given by (3.5) is sharp since equality is attained for every monomial matrix $A$. 
Remark 3.7 The inequality (3.5) was already proven in Theorem 1 of [10], see also [7, p.145, Theorem 1.7], using essentially the same techniques we do. The inequality (3.5) was also proven in Theorem 8 of [11], and is weaker than [9, Formula (25)].

The following theorem states the best upper bound on $\left|\prod_{i=1}^{k} \lambda_{i}(A)\right|$ in terms of the $l_{2}$ norms of rows and columns of $A$ that can be derived from Theorem 2.8 and from the inequalities (2.23) and (2.28).

Theorem 3.8 Let $A \in \mathbb{C}^{n n}$. Then

$$
\left|\prod_{i=1}^{k} \lambda_{i}(A)\right| \leq\left(\frac{n}{k}\right)^{\frac{k}{2}} \min \left\{\max _{\substack{\alpha \subseteq\{1, \ldots, n\} \\|\alpha|=k}} \prod_{i \in \alpha}\left\|\operatorname{col}_{i}(A)\right\|_{2}, \max _{\substack{\alpha \subseteq\{1, \ldots, n\} \\|\alpha|=k}} \prod_{i \in \alpha}\left\|\operatorname{row}_{i}(A)\right\|_{2}\right\} .
$$

Remark 3.10 In order to justify that (3.9) is indeed the best we can derive from Theorem 2.8 and from the inequalities (2.23) and (2.28), we have to show that for $k<n$ we have

$$
\left(\frac{n}{k}\right)^{\frac{k}{2}} \leq\left(\begin{array}{l}
n \\
k
\end{array}\right)(k+1)^{\frac{k-1}{2}}
$$

This follows from a stronger inequality, see Remark 3.19 below.

Remark 3.11 Note that by Theorem 2.8 the inequality (3.9) is strict whenever $A$ is a nonsingular matrix.

Remark 3.12 The upper bound on $\left|\prod_{i=1}^{k} \lambda_{i}(A)\right|$ given by (3.9) is sharp. It is easy to check that, as in the proof of the equality case in Theorem 2.8 , equality holds for a positive semidefinite $n \times n$ matrix $A$ such that the positive semidefinite matrix $B=A^{2}$ has diagonal elements all equal to 1 and where the eigenvalues of $B$ are $\frac{n}{k}$ with multiplicity $k$ and 0 with multiplicity $n-k$.

Remark 3.13 Inequality (3.9) is a generalization of Hadamard determinant theorem, which is the special case of $(3.9)$ where $k=n$.

Remark 3.14 The special case of $(3.9)$ where $k=n-1$, that is, the inequality

$$
\left|\prod_{i=1}^{n-1} \lambda_{i}(A)\right| \leq\left(1+\frac{1}{n-1}\right)^{\frac{n-1}{2}} \min \left\{\max _{k} \prod_{\substack{j=1 \\ j \neq k}}^{n}\left\|\operatorname{row}_{j}(A)\right\|_{2}, \max _{k} \prod_{\substack{j=1 \\ j \neq k}}^{n}\left\|\operatorname{col}_{j}(A)\right\|_{2}\right\},
$$

follows from [四, Theorem 1]. 
Remark 3.15 Another result in Theorem 8 of $[11$ could be stated as

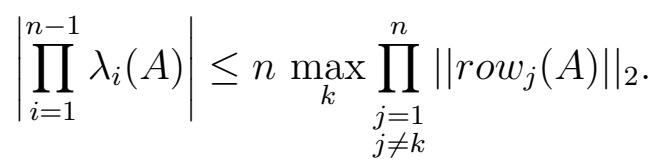

This inequality follows from the special case of our inequality (3.9) where $k=n-1$. It is, in fact, weaker than our result since

$$
\left(1+\frac{1}{n-1}\right)^{\frac{n-1}{2}}<\sqrt{e}<n \quad(n>1) .
$$

Remark 3.16 The upper bounds on $\left|\prod_{i=1}^{k} \lambda_{i}(A)\right|$ given by (3.5) and by (3.9) are not comparable. The bound given by (3.5) is better, for example, in the case of a monomial matrix $A$, since in such a case the $l_{1}$ norm and the $l_{2}$ norm of the rows (and columns) of $A$ are the same. On the other hand, if $A$ is an $n \times n$ complex matrix satisfying $\left|a_{i j}\right|=1, i, j=1, \ldots, n$ and $A A^{*}=n I$ then the $l_{1}$ norm of any row and column of $A$ is equal to $n$, while the $l_{2}$ norm of any row and column of $A$ is equal to $\sqrt{n}$. Therefore, the left hand side of (3.5) becomes $n^{k}$ while the left hand side of (3.9) becomes $\frac{n^{k}}{k^{\frac{k}{2}}}$, which is a better upper bound.

The following theorem states the best upper bound on $\left|\prod_{i=1}^{k} \lambda_{i}(A)\right|$ in terms of the $l_{\infty}$ norms of rows and columns of $A$ that can be derived from Theorem 2.12 and from the inequalities (2.24) and (2.26).

Theorem 3.17 Let $A \in \mathbb{C}^{n n}$. Then

$$
\begin{gathered}
\left|\prod_{i=1}^{k} \lambda_{i}(A)\right| \leq \\
\left(\frac{n}{k}\right)^{\frac{k}{2}} \sqrt{n}{ }^{k} \min \left\{\max _{\substack{\alpha \subseteq\{1, \ldots, n\} \\
|\alpha|=k}} \prod|| \operatorname{col}_{i}(A)\left\|_{\infty}, \max _{\substack{\alpha \subseteq\{1, \ldots, n\} \\
|\alpha|=k}} \prod_{i \in \alpha}\right\| \operatorname{row}(A) \|_{\infty}\right\} .
\end{gathered}
$$

Remark 3.19 In order to justify that (3.18) is indeed the best we can derive from Theorem 2.12 and from the inequalities (2.24) and (2.26), we have to show that for $k<n$ we have

$$
\left(\frac{n}{k}\right)^{\frac{k}{2}} \sqrt{n}^{k} \leq\left(\begin{array}{l}
n \\
k
\end{array}\right)(k+1)^{\frac{k-1}{2}}
$$


or, equivalently,

$$
h(k, n)=\frac{\left(\frac{n}{k}\right)^{\frac{k}{2}} \sqrt{n}^{k}}{\left(\begin{array}{l}
n \\
k
\end{array}\right)(k+1)^{\frac{k-1}{2}}}=\frac{n^{k}}{\sqrt{k}^{k} \sqrt{k+1}^{k-1}\left(\begin{array}{l}
n \\
k
\end{array}\right)} \leq 1 .
$$

Note that

$$
h(1, n)=1, \quad \forall n .
$$

Since $k \leq n-1$ we have $(2 n+1) k<2 n^{2}$, which is equivalent to

$$
\frac{k+2}{k}>\frac{(n+1)^{2}}{n^{2}}
$$

or

$$
\frac{n \sqrt{k+2}}{\sqrt{k}(n+1)}>1
$$

It is easy to check that

$$
\frac{h(k, n)}{h(k+1, n+1)}=\left(\frac{n \sqrt{k+2}}{\sqrt{k}(n+1)}\right)^{k} .
$$

It now follows from (3.21), (3.22) and (3.23) that for every $k$ and $n, k \leq n$, we have $h(k, n) \leq 1$, proving $(3.20)$.

Remark 3.24 By (2.21), the upper bound on $\left|\prod_{i=1}^{k} \lambda_{i}(A)\right|$ given by (3.18) follows from the one given by (3.9). The bounds given by (3.18) and by (3.5) are not comparable. The bound given by (3.5) is better, for example, in the case of a monomial matrix $A$, since in such a case the $l_{1}$ norm and the $l_{\infty}$ norm of the rows (and columns) of $A$ are the same. On the other hand, if $A$ is an $n \times n$ complex matrix satisfying $\left|a_{i j}\right|=1, i, j=1, \ldots, n$ and $A A^{*}=n I$ then the $l_{1}$ norm of any row and column of $A$ is equal to $n$, while the $l_{\infty}$ norm of any row and column of $A$ is equal to 1 . Therefore, the left hand side of (3.5) becomes $n^{k}$ while the left hand side of (3.18) becomes $\frac{n^{k}}{k^{\frac{k}{2}}}$, which is a better upper bound.

Our final remark refers to products of smallest (moduli of) eigenvalues of a given $n \times n$ matrix $A$.

Remark 3.25 Since $\prod_{i=k+1}^{n} \lambda_{i}(A)=\frac{\operatorname{det}(A)}{\prod_{i=1}^{k} \lambda_{i}(A)}$, it follows that all the results of this section on upper bounds on the products $\left|\prod_{i=1}^{k} \lambda_{i}(A)\right|$ of $k$ largest eigenvalues of $A$ yield, whenever $A$ is nonsingular, lower bounds on the products $\left|\prod_{i=k+1}^{n} \lambda_{i}(A)\right|$ of $n-k$ smallest eigenvalues of $A$. 


\section{References}

[1] A.C. Aitken, Determinants and Matrices, 9th edn., Oliver and Boyd, Edinburgh, 1956.

[2] F.L. Bauer, J. Stoer and C. Witzgall, Absolute and monotonic norms, Numer. Math. 3: 257-264 (1961).

[3] G.H. Hardy, J.E. Littlewood and G. Polya, Inequalities, Cambridge University Press, 1952.

[4] Y.P. Hong and C.-T. Pan, A lower bound for the smallest singular value, Lin. Alg. Appl. 172: 27-32 (1992).

[5] A. Horn, Doubly stochastic matrices and the diagonal of a rotation matrix, Amer. J. Math. 76: 620-630 (1954).

[6] R.A. Horn and C.R. Johnson, Matrix Analysis, Cambridge University Press, 1985.

[7] M. Marcus and H. Minc, A Survey of Matrix Theory and Matrix Inequalities, Allyn and Bacon, Boston, 1964.

[8] L. Mirsky, Matrices with prescribed characteristic roots and diagonal elements, J. London Math. Soc. 33: 14-21 (1958).

[9] A. Ostrowski, Note on a theorem by Hans Schneider, J. London Math. Soc. 37: 225234 (1962).

[10] H. Schneider, An inequality for latent roots of a matrix applied to determinants with dominant main diagonal, J. London Math. Soc. 28: 8-20 (1953).

[11] Z. Shi and B. Wang, Determinants, eigenvalues of certain classes of matrices, and some estimates on bounds for the condition number, Acta Mathematicae 15 (3): 326341 (1965). (in Chinese)

[12] R.A. Smith, The condition numbers of the matrix eigenvalue problem, Numer. Math. 10: 232-240 (1967). 